\title{
Colorectal Inflammatory Pseudopolyps: A Retrospective Analysis of 70 Patients
}

\author{
Ankit V. Gandhi, Sajjad M. Malik, Juan P. Palazzo* \\ Department of Pathology, Thomas Jefferson University Hospital, Philadelphia, PA, USA \\ Email: ${ }^{*}$ juan.palazzo@jefferson.edu
}

Received 8 May 2014; revised 8 June 2014; accepted 28 June 2014

Copyright (C) 2014 by authors and Scientific Research Publishing Inc.

This work is licensed under the Creative Commons Attribution International License (CC BY). http://creativecommons.org/licenses/by/4.0/

(c) (i) Open Access

\begin{abstract}
Introduction: Inflammatory pseudopolyps (IPs) are a well-recognized entity in patients with inflammatory bowel disease (IBD), most likely developing from long-standing chronic inflammation. Similarly, IPs have been associated with ischemic and infectious colitis, intestinal ulcers, and mucosal anastomoses. This study aimed to analyze inflammatory pseudopolyps without a history of these known associated pathologies. Materials and Methods: A database search was conducted for patients who underwent biopsies at Thomas Jefferson University Hospital from 2003-2013 for the presence of colorectal IPs. Exclusion criteria consisted of patients with a history of IBD, mucosal anastomoses, ischemic and infectious colitis. Spatial and temporal associations between colonic pathologies and IPs were assessed via Fisher's exact and chi-square test, respectively. Results: Seventy-five polyps from 70 patients fulfilled the database search criteria. Forty-one pseudopolyps (55\%) arose from the rectosigmoid region. Twenty-two patients had no associated colon pathology (31\%); 35 patients had epithelial polyps (50\%), such as tubular adenomas, serrated adenomas, and hyperplastic polyps; 10 patients had colonic adenocarcinoma (16\%), and 18 patients had diverticulosis (26\%). Epithelial polyps were significantly associated with IPs in the same region. However, diverticulosis was independent of IPs in regard to space and time. Conclusion: Colorectal inflammatory pseudopolyps may develop sporadically in up to one third of the cases while others frequently arise in the background of non-IBD colonic pathology. The increased presence of these polyps in the left colon raises the possibility that a subset of them may arise in predisposed mucosa. These polyps need to be differentiated from other morphologically similar colonic polyps.
\end{abstract}

\section{Keywords}

Inflammatory Pseudopolyps, Non-Inflammatory Bowel Disease, Sporadic, Pathologies

\footnotetext{
${ }^{*}$ Corresponding author.
} 


\section{Introduction}

Inflammatory pseudopolyps (IPs) often develop in response to chronic inflammation in patients with inflammatory bowel disease (IBD). These polyps are residual mucosal islands of stroma, epithelium, and inflammatory cells found between regions of repeated mucosal ulceration and regeneration. While not specific to ulcerative colitis, IPs often occur in clusters throughout inflamed areas of the colon. Even though IPs are non-neoplastic, they are known to be associated with surrounding dysplasia in the setting of IBD. Likewise, IPs have been associated with ischemic colitis, neonatal necrotizing enterocolitis, infectious colitis, and are frequently seen in the borders of intestinal ulcers and mucosal anastomoses [1].

The terms inflammatory polyp and pseudopolyp have been used interchangeably, and for all practical purposes are the same entity. Pseudopolyps may indicate polypoid mucosal formations following an acute colitis, whereas inflammatory polyps represent polyps formed after a chronic pattern of injury [2]. The two entities have been lumped together to form the term "inflammatory pseudopolyp”, which encompasses two temporally distinct yet similar pathologic processes. Recently, some have argued that the term "post inflammatory polyp” be used given the unknown context of such polyps on discovery [3]. Nevertheless, given its long use in the literature, such polyps still will be referred to as inflammatory pseudopolyp. To date, very little is known about the development of pseudopolyps outside known colonic pathology. Given the strong association between inflammatory pseudopolyps and known pathological processes such as IBD, the purpose of this study was to examine IPs in patients without a history of these colonic disorders to gain further insight into its development.

\section{Materials and Methods}

\subsection{Database Collection}

A database search was conducted for patients who underwent biopsies at Thomas Jefferson University Hospital from 2003-2013 for the presence of colorectal inflammatory pseudopolyps. Exclusion criteria consisted of patients with a history of inflammatory bowel disease (Crohn's or ulcerative colitis), ischemic colitis, infectious colitis, and mucosal anastomoses. Patient information about any history of colorectal inflammatory pseudopolyps and other pathology diagnoses were collected. The Institutional Review Board of Thomas Jefferson University Hospital approved this study.

\subsection{Statistical Analysis}

Descriptive statistics were calculated for clinicopathological features, which included gender, age, pseudopolyp size, location, and prevalence of other colonic pathology. Pseudopolyps association with colonic pathologies in regard to location was evaluated via a two-sided Fisher's exact test. The temporal relationship between several pathologies and pseudopolyps using diagnosis dates was assessed via chi-square test. All tests of significance were conducted with an alpha value of 0.05 .

\section{Results}

\subsection{Histopathologic Findings}

There is a spectrum of findings seen in pseudopolyps, ranging from hyperplastic and cystically dilated glands to polyps in a predominantly expanded lamina propria (Figure 1) of inflammation, edema, and granulation tissue with few or no glands present (Figure 2). The surface mucosa is frequently ulcerated and lamina propria may contain atypical stromal cells (Figure 3). None of the polyps in our study showed adenomatous changes or evidence of epithelial dysplasia. Interestingly, isolated polyps not associated with pathology of the background mucosa shared identical morphologic features with other non-IBD associated IPs.

\subsection{Patient Characteristics}

Seventy-five pseudopolyps from 70 patients matched from database search criteria. An outline of relevant clinical patient and pseudopolyp information is provided in Table 1 . The average age at the time of surgical resection or biopsy was 61.0 years. The gender distribution was somewhat skewed, with 40 males (57\%) and 30 females (43\%). The IPs ranged in size from $0.1-2.2 \mathrm{~cm}$. The majority of the IPs were located in either the sigmoid 


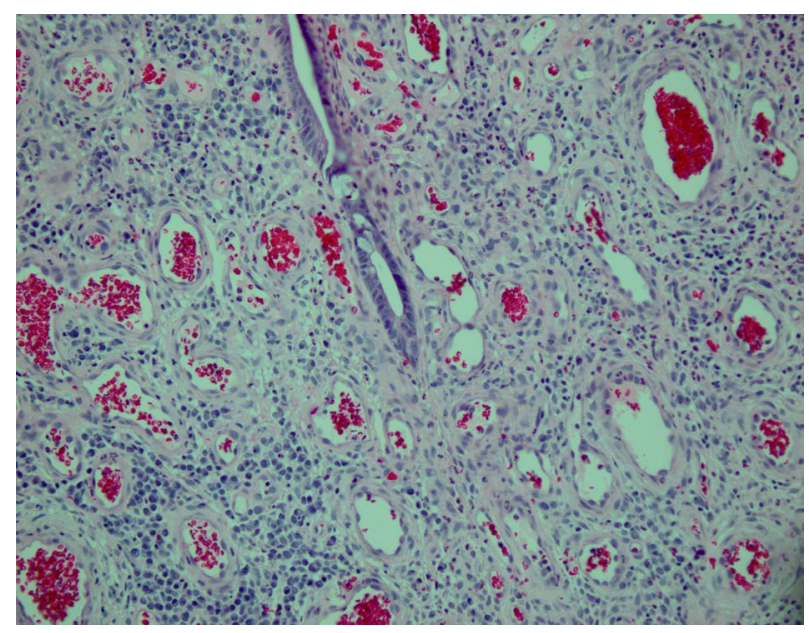

Figure 1. Inflammatory pseudopolyp with an expanded lamina propria and benign reactive glands $(\times 400)$.

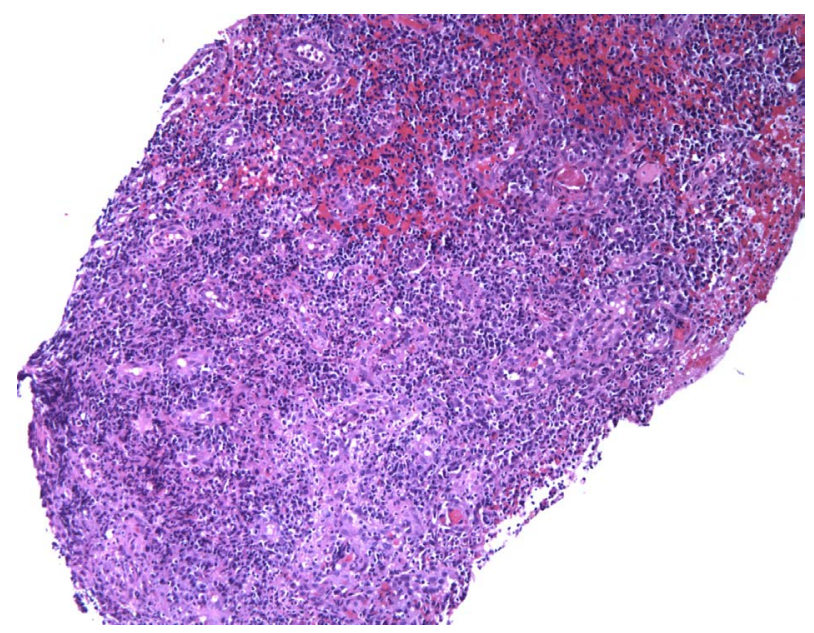

Figure 2. Inflammatory pseudopolyp with ulceration and granulation tissue without glands $(\times 200)$.

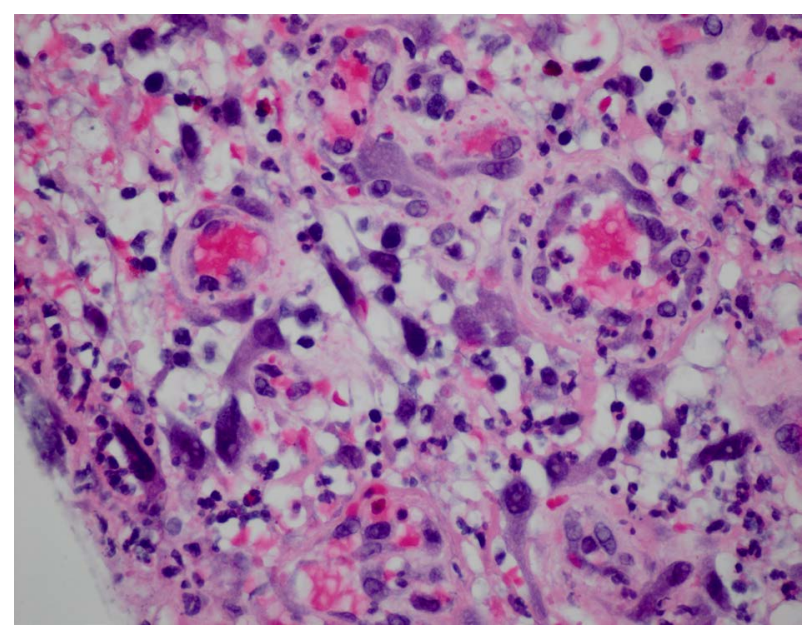

Figure 3. Inflammatory pseudopolyp with granulation tissue and benign atypical stromal cells $(\times 400)$. 
Table 1. Clinical characteristics of patients and inflammatory pseudopolyps.

\begin{tabular}{|c|c|}
\hline Clinical Parameter & \\
\hline \multicolumn{2}{|l|}{ Gender, n (\%) } \\
\hline Male & $40(57.1)$ \\
\hline Female & 30 (42.9) \\
\hline Age, mean (SD) & $61.0(12.3)$ \\
\hline Pseudopolyp size range (cm) & $0.1-2.2$ \\
\hline \multicolumn{2}{|l|}{ Location, n (\%) } \\
\hline Cecum & $5(6.7)$ \\
\hline Ascending & $3(4.0)$ \\
\hline Hepatic flexure & $3(4.0)$ \\
\hline Transverse & $16(21.3)$ \\
\hline Splenic flexure & $3(4.0)$ \\
\hline Descending & $4(5.3)$ \\
\hline Sigmoid & $23(30.7)$ \\
\hline Rectum & $18(24.0)$ \\
\hline \multicolumn{2}{|l|}{ Colonic pathology, n (\%) } \\
\hline None & $22(31.4)$ \\
\hline Epithelial polyp $^{1}$ & $35(50.0)$ \\
\hline Colonic adenocarcinoma & $10(14.3)$ \\
\hline Diverticulosis & $18(25.7)$ \\
\hline Other $^{2}$ & $2(2.9)$ \\
\hline
\end{tabular}

(31\%), rectal (24\%), or transverse (21\%) regions. In regards to the presence of other colonic pathology, 35 patients had epithelial polyps (50\%), 10 patients had colonic adenocarcinoma (16\%), 18 patients had diverticulosis (26\%), one patient had graft-versus-host disease, and one patient had follicular lymphoma. Interestingly, 22 patient cases (31\%) demonstrated sporadic pseudopolyp formation without any other colonic pathology since follow-up. There was overlap of pathologies - seven patients had a history of epithelial polyps and colonic adenocarcinoma, whereas 10 patients had a history of epithelial polyps and diverticulosis. Similarly, five patients had a history of IPs, three of which having recurred in the same location as that of the initial pseudopolyp diagnosis.

\subsection{Spatial Association between Pseudopolyps and Colonic Pathology}

The stratification of colonic pathology by location in relation to IPs is shown in Table 2. The majority of the epithelial polyps (71\%) were found to be significantly associated in the same region as that of pseudopolyps (p < 0.05). While $90 \%$ of colonic adenocarcinomas were located in close proximity to IPs, there was not a significant association probably due to a limited sample size $(p=0.1449)$. In contrast, diverticulosis was distributed more evenly in same and different regions to that of IPs $(\mathrm{p}<0.05)$.

\subsection{Temporal Association between Pseudopolyps and Colonic Pathology}

Category counts of colonic pathology were subdivided into time periods relative to the diagnosis of IPs, as seen in Table 3. The majority of the epithelial polyps (66\%) and colonic adenocarcinomas (80\%) were diagnosed 
Table 2. Background colorectal pathology stratified by location relative to that of inflammatory pseudopolyps.

\begin{tabular}{cccc}
\hline \multirow{2}{*}{ Colonic pathology, n (\%) } & \multicolumn{3}{c}{ Location relative to pseudopolyp } \\
\cline { 2 - 4 } & Same & Different & p-value \\
\hline Epithelial polyp & $25(71.4)$ & $10(28.6)$ & $0.0366^{*}$ \\
Colonic adenocarcinoma & $9(90.0)$ & $1(10.0)$ & 0.1449 \\
Diverticulosis & $8(44.4)$ & $10(55.6)$ & $0.0386^{*}$ \\
Other & $2(100.0)$ & 0.0 & - \\
Total & 44 & 22 & - \\
\hline
\end{tabular}

Statistical significance was determined by Fisher's exact test. ${ }^{*} \mathrm{p}<0.05$.

Table 3. Background colorectal pathology stratified temporally by diagnosis date relative to that of inflammatory pseudopolyps.

\begin{tabular}{ccccc}
\hline \multirow{2}{*}{ Colonic pathology, n (\%) } & \multicolumn{3}{c}{ Diagnosis date relative to pseudopolyp } \\
\cline { 2 - 4 } & Before & Same & After & p-value \\
\hline Epithelial polyp & $23(65.7)$ & $7(20.0)$ & $5(14.3)$ & 0.3985 \\
Colonic adenocarcinoma & $8(80.0)$ & $2(20.0)$ & $0(0.0)$ & 0.2314 \\
Diverticulosis & $5(27.8)$ & $8(44.4)$ & $5(27.8)$ & $0.0079^{* *}$ \\
Other & $2(100.0)$ & $0(0.0)$ & $0(0.0)$ & - \\
Total & 38 & 17 & 10 & - \\
\hline
\end{tabular}

Statistical significance was determined by chi-square test. ${ }^{* *} \mathrm{p}<0.01$.

prior to the discovery of pseudopolyps. However, diverticulosis had a more even distribution of diagnosis dates ( $<<0.01$ ), which implies that diverticulosis is independent of the onset of pseudopolyps.

\section{Discussion}

Inflammatory pseudopolyps are one of the most common complications found in inflammatory bowel disease and have been associated with other pathological phenomenon, such as ischemic and infectious colitis [1] [2]. The histologic features have been well characterized as polypoid, edematous, regenerative mucosal glands resulting from ulcerated areas with granulation tissue [4]. The aim of this study was to evaluate pseudopolyps barring the most common associated pathological conditions, and we found a significant proportion of these cases (31\%) arise sporadically without the presence of any other colonic pathology. Since these sporadic polyps share identical morphologic features with other non-IBD associated IPs, it's interesting to speculate that these patients may have an inherent defect in their mucosa predisposing them to these inflammatory lesions.

The fact that IPs were seen more commonly in the left colon suggests that they may arise in the background of a mucosa prone to their formation. Interestingly, these IPs were not associated with prolapse or showed the classic histologic features of prolapse associated polyps. None of these patients had endoscopic evidence of prolapse; nonetheless, we cannot exclude the possibility that the mucosa of the left colon is more prone to the formation of these polyps and that prolapse may play a role in their formation.

At the same time, a significant spatial association between epithelial polyps and pseudopolyps was evident. This finding suggests that colonic changes from epithelial polyps may aid in the development of pseudopolyps and vice-versa. Since many patients had polyps, such as tubular adenomas, tubulovillous adenomas, and serrated adenomas, and $14 \%$ had colonic adenocarcinomas, there is a possibility that these patients have an inherent predisposition in their mucosa to develop inflammatory pseudopolyps. However, the temporal association that would imply that epithelial polyps serve as risk factors for the development of pseudopolyps later on was not significant. By increasing the patient database while still adhering to exclusion criteria may provide the addi- 
tional statistical power needed to make such a conclusion. On another note, the majority of cases with epithelial polyps and colonic adenocarcinoma developed IPs after these diagnoses, raising the suspicion that increased endoscopic surveillance, instrumentation, and therapy may play a role in pseudopolyposis formation in the background of a predisposed mucosa. Unfortunately, this information was not available for analysis. While the relationship between epithelial polyps and pseudopolyps remains unclear, the significant associations of diverticulosis and IPs insinuate that both pathologic conditions are independent of each other in regards to space and time. However, the role of previous episodes of diverticulitis is not entirely clear, especially since this information was not readily available. Only five patients could potentially have had diverticulitis propagating IPs. Given that diverticular disease was uniformly distributed across space and time, the impact of diverticulosis and possibly diverticulitis on the development of IPs appears to be minimal.

IPs are usually not a diagnostic challenge for pathologists, but they need to be differentiated from other polyps, such as inflamed tubular adenomas and juvenile-type polyps. Inflamed TAs are characterized by dysplastic glands while juvenile type polyps show cystically dilated hyperplastic glands without the significant inflammation of the lamina propria as seen in IPs. However, these polyps may have overlapping features, and it maybe difficult to differentiate between them. Likewise, IPs prominent inflammation may elicit the appearance of a highly reactive epithelial component as single glands or atypical stromal cells that can be mistaken for dysplasia. These reactive changes should not be overly interpreted as such. Additionally, the atypical stromal cells can resemble dysplastic epithelial cells invading the stroma of the polyp and could lead to a differential diagnosis that includes invasive carcinoma. Some authors have recommended using a cytokeratin immunohistochemical stain to rule out these cells being epithelial in nature. In our view and experience, there is no need to perform such stains in most cases.

In the differential diagnosis of inflammatory pseudopolyps, one must also consider the inflammatory fibroid polyp, a separate entity altogether. It is a rare, benign lesion of the gastrointestinal tract, which shares some histologic findings to IPs [5]. Inflammatory fibroid polyps, however, occur much more frequently in the upper GI tract, including the stomach and small intestine. A distinguishing factor is the predominant cell type in the inflammatory fibroid polyp - the fibroblast or myofibroblast-like cell, which is diffusely positive for vimentin and negative for CD117 [6]. The etiology of inflammatory fibroid polyps is unknown, but some consider them to develop from an exaggerated response to trauma, allergy, foreign body, infectious agents, vasoactive substances, or a colonic motility disorder [7]-[10]. It is possible that one or a combination of these factors may be responsible for the development of inflammatory pseudopolyps as well.

Lastly, mucosal prolapsed-induced polyps in the left colon and rectum may be confused with IPs. These polyps have a variable amount of mixed inflammation with granulation tissue and fibrin covering the surface, but unlike pseudopolyps, typically show a hyperplastic, polyp-like architecture with fibromuscular hypertrophy that radially extends into the lamina propria [11]-[13].

\section{Conclusion}

To our knowledge, this is the first study to describe the clinicopathological features of IPs in non-IBD patients. In this study, the majority of these patients developed pseudopolyps in the rectosigmoid region. Nearly a third of patients with pseudopolyps did not have a history of any other colonic pathology since follow-up. Interestingly, sporadic polyps shared identical morphologic features with IPs with colonic pathologies, which included epithelial polyps, colonic adenocarcinoma, and diverticulosis. Lastly, epithelial polyps correlated with inflammatory pseudopolyps with respect to location, while our findings suggested that diverticulosis is independent of pseudopolyps in time and space. Although IPs increasingly are becoming a more frequent diagnosis, not much is known regarding its pathogenesis. Because of this, we hope to see future studies elucidating the clinical significance of inflammatory pseudopolyps.

\section{References}

[1] Iofel, E., Kahn, E., Lee, T. and Chawla, A. (2000) Inflammatory Polyps after Necrotizing Enterocolitis. Journal of Pediatric Surgery, 35, 1246-1247. http://dx.doi.org/10.1053/jpsu.2000.8763

[2] Levine, D.S., Surawicz, C.M., Spencer, G.D., Rohrmann, C.A. and Silverstein, F.E. (1986) Inflammatory Polyposis Two Years after Ischemic Colon Injury. Digestive Diseases and Sciences, 31, 1159-1167. http://dx.doi.org/10.1007/BF01300268 
[3] Quirke, P., Risio, M., Lambert, R., Karsa, L. and Vieth, M. (2011) Quality Assurance in Pathology in Colorectal Cancer Screening and Diagnosis-European Recommendations. Virchows Archiv, 458, 1-10. http://dx.doi.org/10.1007/s00428-010-0977-6

[4] Christos, P., Konstantinos, F., Kanelina, B., Afrodite, P., Stilianos, A. and Hatzitheoharis, G. (2006) Colonic Obstruction Due to Localized Giant Pseudopolyposis Complicating Ulcerative Colitis. The Internet Journal of Surgery, 8.

[5] Vaněk, J. (1949) Gastric Submucosal Granuloma with Eosinophilic Infiltration. The American Journal of Pathology, 25, 397.

[6] Stolte, M. and Finkenzeller, G. (1990) Inflammatory Fibroid Polyp of the Stomach. Endoscopy, 22, 203. http://dx.doi.org/10.1055/s-2007-1012848

[7] Shalom, A., Wasserman, I., Segal, M. and Orda, R. (2000) Inflammatory Fibroid Polyp and Helicobacter Pylori. Aetiology or Coincidence? European Journal of Surgery, 166, 54-57.

[8] Sen, G.R. (2001) Inflammatory Fibroid Polyps of the Gastrointestinal Tract. Pathologe, 22, 333-338. http://dx.doi.org/10.1007/s002920100473

[9] Makhlouf, H.R. and Sobin, L.H. (2002) Inflammatory Myofibroblastic Tumors (Inflammatory Pseudotumors) of the Gastrointestinal Tract: How Closely Are They Related to Inflammatory Fibroid Polyps? Human Pathology, 33, 307315. http://dx.doi.org/10.1053/hupa.2002.32213

[10] Merkel, I.S., Rabinovitz, M. and Dekker, A. (1992) Cecal Inflammatory Fibroid Polyp Presenting with Chronic Diarrhea. Digestive Diseases and Sciences, 37, 133-136. http://dx.doi.org/10.1007/BF01308356

[11] Chetty, R., Bhathal, P. and Slavin, J. (1993) Prolapse-Induced Inflammatory Polyps of the Colorectum and Anal Transitional Zone. Histopathology, 23, 63-67. http://dx.doi.org/10.1111/j.1365-2559.1993.tb01184.x

[12] Corman, M.L. (1985) Rectal Prolapse in Children. Diseases of the Colon \& Rectum, 28, 535-539. http://dx.doi.org/10.1007/BF02554107

[13] Groff, D.B. and Nagaraj, H.S. (1990) Rectal Prolapse in Infants and Children. The American Journal of Surgery, 160, 531-532. http://dx.doi.org/10.1016/S0002-9610(05)81022-0 
Scientific Research Publishing (SCIRP) is one of the largest Open Access journal publishers. It is currently publishing more than 200 open access, online, peer-reviewed journals covering a wide range of academic disciplines. SCIRP serves the worldwide academic communities and contributes to the progress and application of science with its publication.

Other selected journals from SCIRP are listed as below. Submit your manuscript to us via either submit@scirp.org or Online Submission Portal.
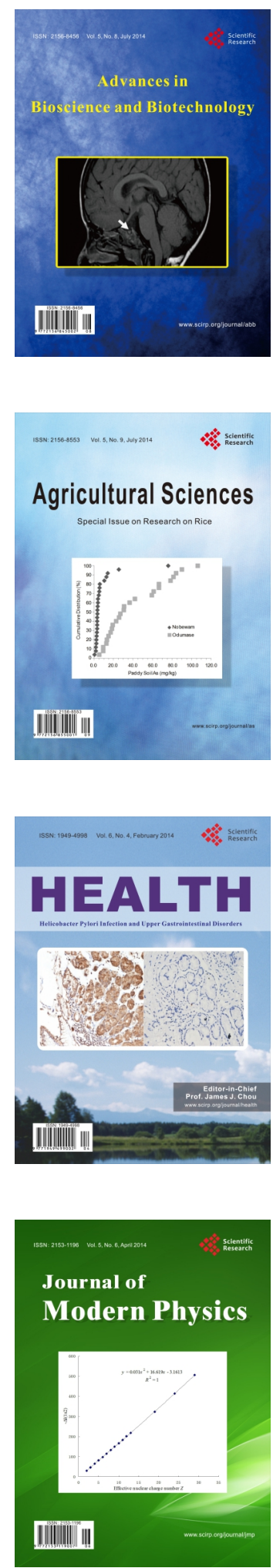
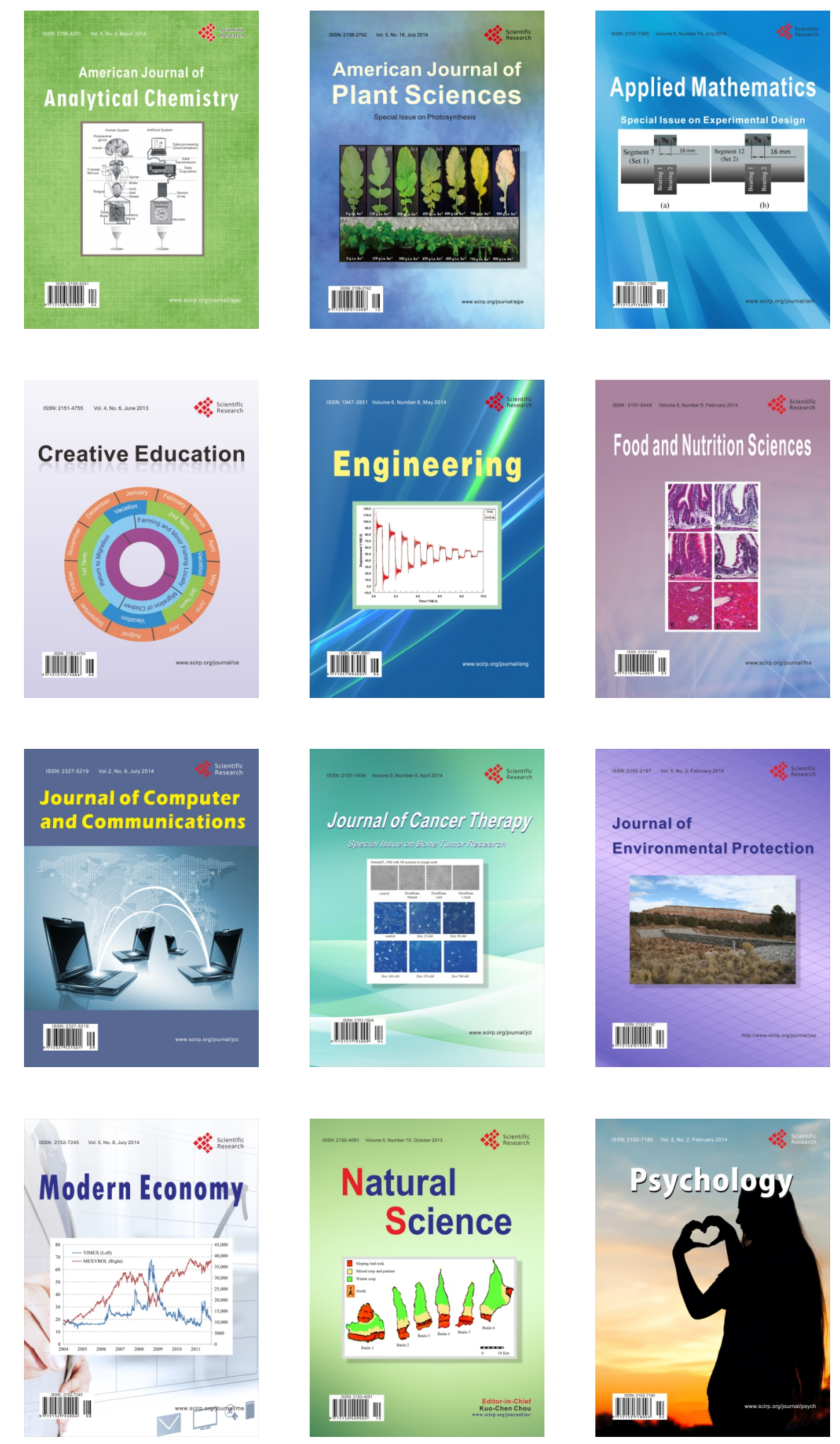